\title{
Hybrid - nicht nur im Auto
}

Das Wort Hybrid hat in den vergangenen Jahren vor allem im Zusammenhang mit Autos öffentliche Bekanntheit erlangt. Es ging dabei um Fahrzeuge, die von einem Verbrennungs- und einem Elektromotor angetrieben werden. Hybride Wertschöpfung meint dagegen, dass Unternehmen nicht nur zum Beispiel mit dem Verkauf von Produkten Gewinne erwirtschaften, sondern zusätzlich etwa mit passenden Beratungsoder Service-Dienstleistungen. Das ist Alltag in deutschen Unternehmen, unter anderem in der Automobilindustrie.

Trotzdem ist der Begriff weit davon entfernt, allgemein bekannt zu sein. Die meisten Firmen verkaufen heute neben den

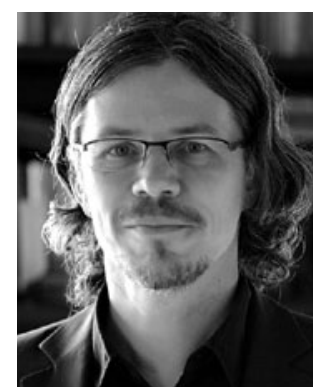

Peter Pagel Chefredakteur

Produkten, die sie herstellen, zusätzliche Dienstleistungen. Das Spektrum reicht dabei vom klassischen Wartungsvertrag bis hin zur Unternehmensberatung. Begleitet wird beispielsweise der gesamte Lebenszyklus einer Maschine, von der Planung über die Inbetriebnahme bis hin zum Recycling am Ende der Produktlebensdauer. Sinnvoll sind diese zusätzlichen Angebote schon deshalb, weil gerade Investitionsgüter nur sehr selten gekauft und dann über Jahrzehnte verwendet werden. Hybride Wertschöpfung sorgt dann für gleichmäßigeren Umsatz. Zudem kann über den intensiveren Kontakt eine stärkere Kundenbindung gefördert werden. Hybride Wertschöpfung lohnt sich also in mehrfacher Weise.

Herzliche Grüße

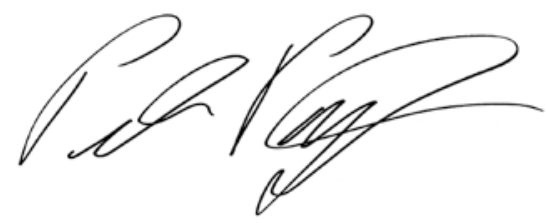

Peter Pagel 